\title{
Resveratrol analogue, HS-1793, induces apoptotic cell death and cell cycle arrest through downregulation of AKT in human colon cancer cells
}

\author{
DONG HWAN KIM ${ }^{1}$, MIN JEONG KIM ${ }^{1}$, BOKYUNG SUNG ${ }^{1}$, HONGSUK SUH ${ }^{2}$, \\ JEE H. JUNG ${ }^{1}$, HAE YOUNG CHUNG ${ }^{1}$ and NAM DEUK KIM ${ }^{1}$ \\ ${ }^{1}$ Department of Pharmacy, Molecular Inflammation Research Center for Aging Intervention, ${ }^{2}$ Department of Chemistry and \\ Chemistry Institute for Functional Materials, Pusan National University, Busan 46241, Republic of Korea
}

Received June 1, 2016; Accepted October 26, 2016

DOI: $10.3892 /$ or.2016.5219

\begin{abstract}
Resveratrol, a polyphenolic compound, is a naturally occurring phytochemical and is found in a variety of plants, including grapes, berries and peanuts. It has gained much attention for its potential anticancer activity against various types of human cancer. However, the usefulness of resveratrol as a chemotherapeutic agent is limited by its photosensitivity and metabolic instability. In this study the effects of a synthetic analogue of resveratrol, HS-1793, on the proliferation and apoptotic cell death were investigated using HCT116 human colon cancer cells. Although this compound has been reported to have anticancer activities in several human cancer cell lines, the therapeutic effects of HS-1793 on human colon cancer and its mechanisms of action have not been extensively studied. HS-1793 inhibited cell growth and induced apoptotic cell death in a concentration-dependent fashion. Induction of apoptosis was determined by morphological changes, cleavage of poly(ADP-ribose) polymerase, alteration of $\mathrm{Bax} / \mathrm{Bcl}-2$ expression ratio, and caspase activations. Flow cytometric analysis revealed that HS-1793 induced $\mathrm{G} 2 / \mathrm{M}$ arrest in the cell cycle progression in HCT116 cells. Furthermore, HS-1793 showed more potent anticancer effects in several aspects than resveratrol in HCT116 cells. In addition, HS-1793 suppressed Akt and the phosphatidylinositol-3 kinase/Akt inhibitor LY294002 was found to enhance its induction of apoptosis. Thus, these findings suggest that HS-1793 have potential as a candidate chemotherapeutic agent against human colon cancer.
\end{abstract}

Correspondence to: Professor Nam Deuk Kim, Department of Pharmacy, College of Pharmacy, Pusan National University, Busandaehag-ro, 63 beon-gil 2, Geumjeong-gu, Busan 46241, Republic of Korea

E-mail: nadkim@pusan.ac.kr

Key words: resveratrol analogue, colon cancer cells, apoptosis, cell cycle

\section{Introduction}

Despite the rapid decline of colorectal cancer (CRC) incidence, due to introduction of CRC screening, in recent years, CRC is still a major cause of incidence and mortality in many countries, especially in more developed ones, though in very recent years its incidence is increasing also in developing areas of the world (1). CRC incidence and mortality has been increasing rapidly in Korea during last few decades (2). Shin et al reported that the age-standardized incidence rate (ASR) of CRC was 27 (per 100,000) in 1999 and increased to 50.2 in 2009 among men (annual percentage changes, 6.6\%) in Korea (3). There are considerable advances in neoadjuvant chemotherapy and improved surgical techniques have been achieved in the past decades, the 5-year survival rate of colon cancer of stage IV was only $8.1 \%$ after treatments (4). In addition, long-term use of chemotherapy can make patient condition worse and develop resistance to chemotherapy. Therefore, novel non-toxic therapeutic agents which are safe, affordable and effective are urgently needed.

Trans-3,4,5'-trihydroxystilbene (resveratrol) (Fig. 1A) is a natural polyphenol and has been shown to prevent tumor formation and development in several cancer types (5-8). This polyphenol has also been shown to kill multiple types of cancer cells (9-11), and to suppress angiogenesis and metastasis in a variety of animal tumor models $(12,13)$. Besides anticancer properties, resveratrol has multiple biological and pharmacologic activities: it has been described as an antidiabetic agent, an anti-aging agent, a platelet aggregation inhibitor, a cardioprotective agent and an anti-inflammatory agent $(14,15)$. Although accumulating evidence on health benefits and anticancer effect of resveratrol exist, the compound has limited its use as a cancer chemoprevention agent, since resveratrol is not a potent cytotoxic agent when compared with other chemotherapeutic agents. Therefore, exposure to high doses of resveratrol is required to induce apoptosis in cancer cells. In addition, the biological activity of resveratrol is limited by its photosensitivity and metabolic instability $(16,17)$.

Multiple approaches are being sought to overcome these limitations, including the design and synthesis of novel structural analogues $(18,19)$. One compound in particular, 
4-(6-hydroxy-2-naphthyl)-1,3-benzenediol (HS-1793), has stronger antitumor effects than resveratrol in most cancer cells tested (20-23). Moreover, HS-1793 overcomes the resistance conferred by Bcl-2 by inducing apoptosis (21) and inhibited hypoxia-induced hypoxia-inducible factor-1 and vascular endothelial growth factor expressions (24). However, the direct molecular target and mode of HS-1793's anticancer mechanism in human colon cancer cells have not been fully clarified. Therefore, this study used human colon cancer HCT116 cells to identify additional molecular mechanisms supporting the antiproliferative and apoptotic effects of HS-1793.

\section{Materials and methods}

Chemicals. Trans-3,4,5'-trihydroxystilbene (resveratrol) was purchased from Sigma-Aldrich Co. (St. Louis, MO, USA). 4-(6-Hydroxy-2-naphthyl)-1,3-benzendiol (HS-1793) was synthesized, kindly supplied by Professor Hongsuk Suh (Pusan National University, Busan, Korea). A 100 mM solution of resveratrol or HS-1793 was prepared in ethanol, and stored in small aliquots at $-20^{\circ} \mathrm{C}$. The stock solution was diluted as needed in cell culture medium. The maximal concentration of ethanol did not exceed $0.1 \%(\mathrm{v} / \mathrm{v})$ in the treatment range, where there was no influence on the cell growth. 3-(4,5-Dimethylthiazol-2-yl)-2,5-diphenyltetrazolium bromide (MTT) was obtained from Amresco LLC (Solon, OH, USA). Antibodies specific for pro-caspase-3, -8 and -9, poly(ADP-ribose) polymerases (PARP), B-cell CLL/lymphoma-2 (Bcl-2), Bcl-2 associated X protein (Bax), cyclin A, cyclin B1, cyclin D1, Cdc2, Cdc25C, cyclin-dependent kinase (CDK) 2, CDK4, CDK6, cytochrome $c$, p38 mitogenactivated protein kinase (MAPK), phospho-extracellular signal-regulated kinase (ERK)1/2 (Thr202/Tyr204), c-Jun N-terminal kinase(JNK), phospho-Akt(Ser473), Akt and glyceraldehyde 3-phosphate dehydrogenase (GAPDH) were obtained from Santa Cruz Biotechnology, Inc. (Dallas, TX, USA). The anti-cleaved-caspase-3, anti-cleaved-caspase-8, phospho-p38 MAPK (Thr180/Tyr182), phospho-JNK (Thr183/Tyr185), and ERK1/2 were purchased from Cell Signaling Technology (Danvers, MA, USA). Antibody against $\beta$-actin and LY294002 were purchased from Sigma-Aldrich Co.

Cell culture and viability assay. HCT116 cells were obtained from American Type Culture Collection (Manassas, VA, USA). Cells were maintained at $37^{\circ} \mathrm{C}$ in humidified $5 \% \mathrm{CO}_{2}$ in RPMI-1640 supplemented with $10 \%$ fetal bovine serum, $100 \mathrm{U} / \mathrm{ml}$ penicillin, and $100 \mu \mathrm{g} / \mathrm{ml}$ streptomycin (both from GE Healthcare Life Sciences, Logan, UT, USA). Cell viability was determined by MTT assay. Cells were seeded in each well of 24-well plate, allowed to adhere overnight, treated with or without various reagents at the indicated concentrations, and then incubated in the dark with $0.5 \mathrm{mg} / \mathrm{ml} \mathrm{MTT}$ at $37^{\circ} \mathrm{C}$ for $2 \mathrm{~h}$. The formazan granules generated by the live cells were dissolved in DMSO, and the absorbance at $540 \mathrm{~nm}$ was monitored using a multi-well reader (Thermo Fisher Scientific, Vantaa, Finland).

Cell proliferation assay. To determine the effect of the test agents on cancer cell proliferation, MTT assay was performed. Briefly, cells were incubated in the presence or absence of

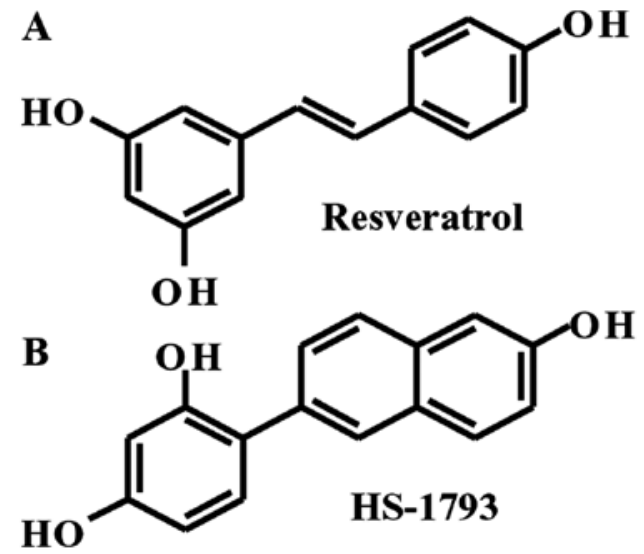

Figure 1. Chemical structures of resveratrol (A) and HS-1793 (B).

the indicated concentration of agents for $0,1,2$ and 4 days. Thereafter, cell growth was measured by MTT assay as described above.

Annexin V/PI staining assay. After treatment with various concentrations of testing agents for $24 \mathrm{~h}$, the cells were trypsinized, washed, and collected. Apoptotic cells were detected using the BD Pharmingen FITC Annexin V apoptosis detection kit (BD Biosciences, San Diego, CA, USA) in accordance with the instruction provided by the manufacturer. A total of 10,000 cells were subsequently collected and analyzed using a flow cytometer (Accuri C6; BD Biosciences, Ann Arbor, MI, USA).

Nuclear staining with Hoechst 33342. The control and treated cells were washed with phosphate-buffered saline (PBS) and fixed with $3.7 \%$ paraformaldehyde in PBS for $10 \mathrm{~min}$ at room temperature. Fixed cells were washed with PBS and stained with $4 \mu \mathrm{g} / \mathrm{ml}$ Hoechst 33342 (Thermo Fisher Scientific, Waltham, MA, USA) for $10 \mathrm{~min}$ at room temperature. And then, the cells were washed with PBS and analyzed by fluorescent microscope.

Flow cytometric analysis for measurement of cell cycle population. The DNA content was measured following the staining of the cells with propidium iodide (PI; Sigma-Aldrich Co.). After treatment with various concentrations of testing agents, the cells were harvested, washed with cold PBS, and further fixed in $70 \%$ ethanol at $-20^{\circ} \mathrm{C}$ overnight. The fixed cells were washed with cold PBS and then stained with cold PI solution $\left(50 \mu \mathrm{g} / \mathrm{ml}\right.$ in PBS) at $37^{\circ} \mathrm{C}$ for $30 \mathrm{~min}$ in the dark. Flow cytometric analysis was performed on an Accuri C6.

Western blot analysis. Cells were harvested and solubilized in whole cell lysis buffer, and the supernatant was collected and protein concentrations were then determined by protein assay reagents (Bio-Rad, Hercules, CA, USA). Subcellular fractions of mitochondria and cytosol were prepared using mitochondria isolation kit for mammalian cells (Thermo Fisher Scientific). Equal amounts of protein extracts were denatured by boiling at $100^{\circ} \mathrm{C}$ for $5 \mathrm{~min}$ in sample buffer (Bio-Rad). Equal amount of protein was subjected to sodium dodecyl sulfate-polyacrylamide gel electrophoresis (SDS-PAGE) and 

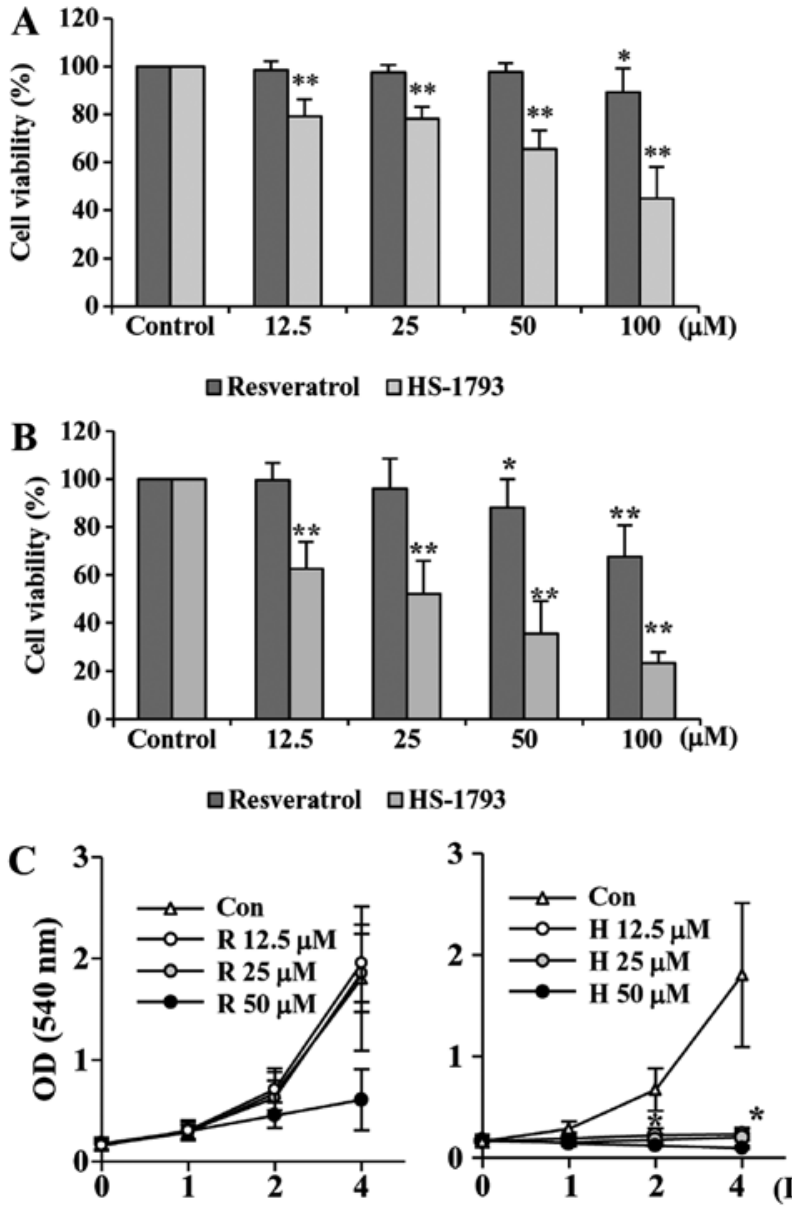

Figure 2. HS-1793 inhibits growth of HCT116 cells. (A and B) The effects of resveratrol and HS-1793 on the proliferation of HCT116 cells. Cells were incubated with increasing concentrations of resveratrol or HS-1793 for (A) $24 \mathrm{~h}$ and (B) $48 \mathrm{~h}$, respectively. Cell viability was determined by MTT assay. Data are the mean \pm SD of three independent experiments. " $p<0.05$ and ${ }^{* *} \mathrm{p}<0.01$ vs. Con. (C) Resveratrol and HS-1793 suppresses HCT116 cell proliferation. Cells were incubated with increasing concentrations $(0,12.5,25$ and $50 \mu \mathrm{M}$ ) of resveratrol (R) or HS-1793 (H) for different time periods. Cel proliferation was then analyzed by the MTT assay as described in Materials and methods. Data are representative of three independent experiments. " $\mathrm{p}<0.05$ and ${ }^{* *} \mathrm{p}<0.01$ vs. Con. Con, vehicle-treated control.

transferred to polyvinylidene fluoride (PVDF) membranes by immunoblotting. Blots were probed with the desired primary antibodies overnight, incubated with horseradish peroxidase (HRP)-conjugated secondary antibodies (Santa Cruz Biotechnology, Inc.), and then visualized using the enhanced chemiluminescence (ECL) detection system (GE Healthcare, Piscataway, NJ, USA).

Statistical analysis. Results are expressed as the mean \pm SD of two or three separate experiments and analyzed by Student's t-test. Means were considered significantly different at ${ }^{*} \mathrm{p}<0.05$ or ${ }^{* *} \mathrm{p}<0.01$.

\section{Results}

HS-1793 suppresses the proliferation of HCT116 cells. To investigate the antiproliferative activities of HS-1793, a synthetic resveratrol analogue, on HCT116 cells, we first performed the cell viability assay. We also used resveratrol for comparison. Treatment cells with resveratrol decreased the cell viability slightly after $24 \mathrm{~h}$, but after $48 \mathrm{~h}$ of treatment the viability was reduced almost by $68 \%$ at $100 \mu \mathrm{M}$ of resveratrol (Fig. 2A). Our data showed that HS-1793 significantly reduced the cell viability concentration- and time-dependently (Fig. 2B). More importantly, HS-1793 exhibited potent growth inhibitory effect when compared to that of resveratrol under same experimental conditions (Fig. 2A and B).

We also determined the effect of resveratrol and HS-1793 on cell proliferation (Fig. 2C). Results indicated that resveratrol showed moderate anti-proliferative effect in HCT116 cells (Fig. 2C, left). However, HS-1793 significantly suppresses proliferation of colon cancer cell line HCT116 (Fig. 2C, right). More importantly, the effects were observed at $12.5 \mu \mathrm{M}$, a concentration at which resveratrol had no significant effect on HCT-116 cell proliferation. The results demonstrate that HS-1793 is more potent than resveratrol in the growth suppression of the human CRC cell line HCT116.

HS-1793 induces apoptosis in HCT116 cells. To investigate the underlying mechanism of growth inhibition observed in the cell viability and cell proliferation assay, we next examined apoptosis effect on HCT116 cells induced by resveratrol and HS-1793 using Annexin V/PI staining as described in Materials and methods section. Cells showed concentration-dependent apoptosis after a $24 \mathrm{~h}$ treatment with resveratrol or HS-1793 (Fig. 3A). The analysis demonstrated that treatment with $50 \mu \mathrm{M}$ resveratrol induced apoptosis in $22.4 \%$, whereas same concentration of HS-1793 induced apoptosis in $31.6 \%$ of HCT116 cells.

In order to determine whether HS-1793 induces morphological changes, one characteristic of apoptosis, Hoechst staining was performed. By Hoechst staining, it was shown that resveratrol and HS-1793 caused chromatin condensation and fragmentation which are typical apoptotic nuclear morphological changes (Fig. 3B). The untreated cells exerted oval nuclear structure, while the cells treated with resveratrol and HS-1793 exhibited evident apoptotic characteristics, including shrinkage and nuclear condensation (Fig. 3B). Compared with resveratrol, there was an evident increase in the number of nuclear-condensed cells following treatment with HS-1793 at the same concentration.

Then we determined the effect of resveratrol and HS-1793 on the levels of apoptosis-related proteins in HCT116 cells. At $100 \mu \mathrm{M}$, HS-1793 effectively induced the reduction of pro-caspase- 8 and pro-caspase-3, whereas resveratrol did not (Fig. 4A). HS-1793 also activated caspase-8 and caspase-3 as indicated by the presence of cleaved caspases. Similar result was observed in cleavage of PARP in HCT116 cells (Fig. 4A). It is noticeable that HS-1793 $(25 \mu \mathrm{M})$ caused the PARP cleavage, whereas resveratrol $(100 \mu \mathrm{M})$ had no significant effect on PARP cleavage in HCT116 cells. However, both resveratrol and HS-1793 had no effect on pro-caspase-9 expression (Fig. 4A). In addition, treatment of HS-1793 slightly downregulated the level of antiapoptotic protein $\mathrm{Bcl}-2$ at high concentration $(100 \mu \mathrm{M})$ while resveratrol further upregulated the level of Bcl-2 (Fig. 4B). In HCT116 cells, the level of apoptosis-promoting protein Bax was induced by both HS-1793 and resveratrol, and a more prominent effect was observed in HS-1793-treated cells (Fig. 4B). These data 

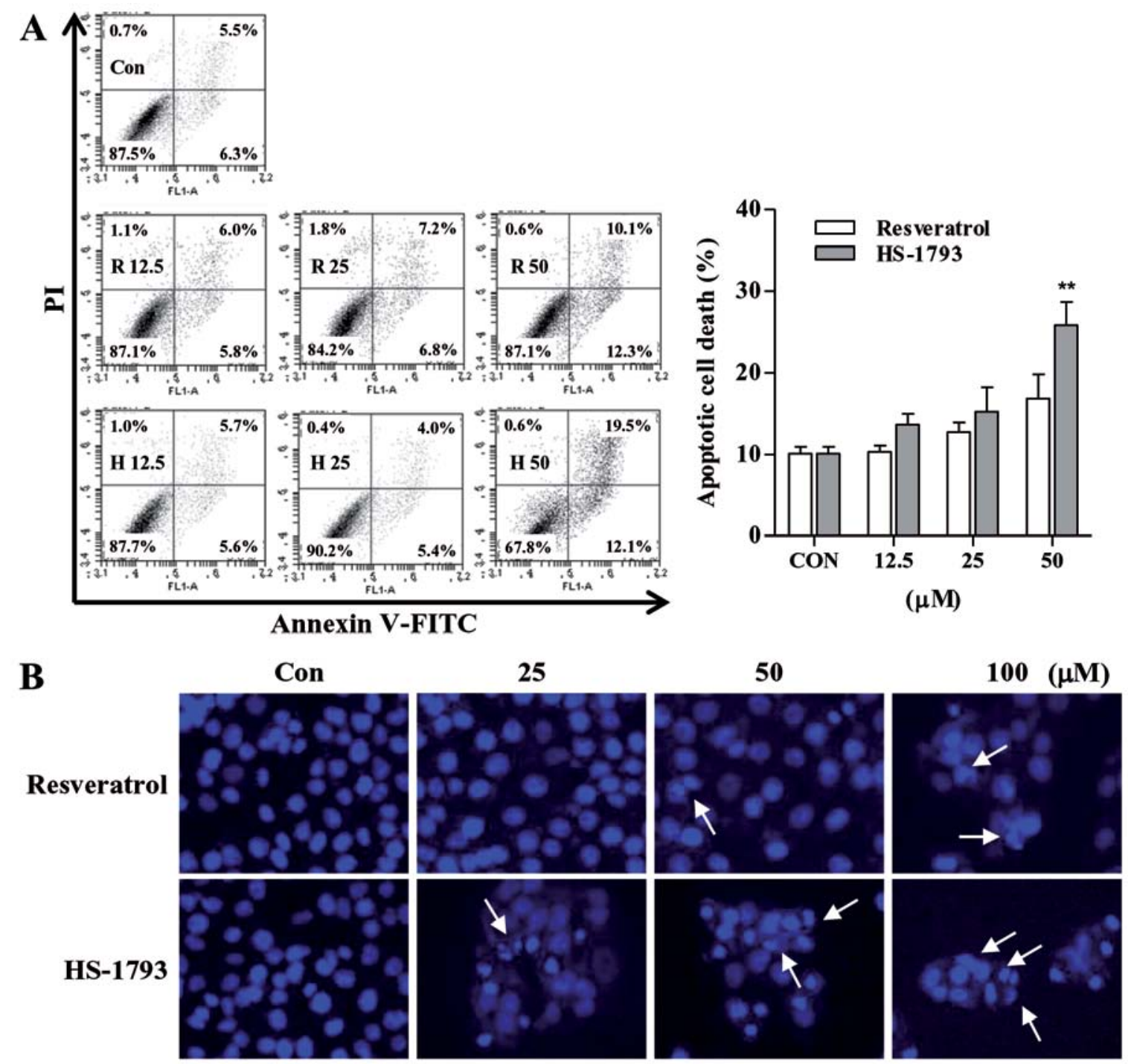

Figure 3. HS-1793 induces apoptotic cell death in HCT116 cells. (A) Induction of apoptosis in HCT116 cells was determined by flow cytometry after treatment with increasing concentrations $(0,12.5,25$ and $50 \mu \mathrm{M})$ of resveratrol (R) or HS-1793 (H) for $24 \mathrm{~h}$. The representative results from three independent experiments are shown (left panel). The graph (right panel) represents percent apoptotic population (Annexin V positive). Data are the mean \pm SD of one of three independent experiments. ${ }^{* *}$ p $<0.01$ vs. Con. (B) Cells were treated with different concentrations of resveratrol or HS-1793 for 24 h, stained with Hoechst 33342 for $10 \mathrm{~min}$ and then observed with a fluorescence microscope (original magnification, $\mathrm{x} 400$ ). The arrow indicates chromatin condensation. Data represent one of three independent experiments. Con, vehicle-treated control. PI, propidum iodide; Con, vehicle-treated control.

suggest that HS-1793 is a more potent inducer of apoptosis than resveratrol.

HS-1793 induces cytochrome c release in HCT116 cells. Mounting evidence suggests that mitochondria play an essential role in apoptosis by releasing apoptogenic effectors such as cytochrome $c$ (25). In order to determine the involvement of the mitochondrial pathway in HS-1793-induced apoptosis in HCT116 cells, we analyzed the cytosolic and mitochondrial levels of cytochrome $c$. The results of western blot analyses demonstrated that both resveratrol and HS-1793 promoted an increase in the release of cytochrome $\mathrm{c}$ from the mitochondria into the cytosol (Fig. 4C). HS-1793 was stronger stimulatory effects on inducing cytochrome c release when compared to that of resveratrol.

HS-1793 promotes G2/M cell cycle arrest in HCT116 cells. To determine whether the growth inhibition by HS-1793 or resveratrol was caused by cell cycle arrest, the cells were incubated with various concentrations of HS-1793 for $24 \mathrm{~h}$. The cells were then fixed, stained and cell cycle populations were determined by flow cytometry. The results showed that HS-1793 induced the accumulation of cells in the G2/M phase in a concentration-dependent manner while $S$ phase arrest was observed in resveratrol-treated cells (Fig. 5). Next, we examined the effect of HS-1793 on the expression of G2/M cell cycle regulators. Cell cycle checkpoints are mainly regulated by several kinds of cyclin-dependent kinase (CDK) complexes. Above all, G2/M transition is largely dependent on cyclin B1/Cdc2 (Cdk1) activity (26). Thus the activity of cyclin $\mathrm{B} 1 / \mathrm{Cdc} 2$ complex is regulated by the positive regulator $\mathrm{Cdc} 25 \mathrm{C}$, and two negative regulators, the protein kinases Weel and Myt1 (27). The western blot results indicated that the expression of $\mathrm{G} 2 / \mathrm{M}$ cell cycle regulatory protein cyclin $\mathrm{B} 1$, $\mathrm{Cdc} 2$ and $\mathrm{Cdc} 25 \mathrm{C}$ decreased by increasing concentration of HS-1793 (Fig. 6A). Resveratrol also downregulated the protein 

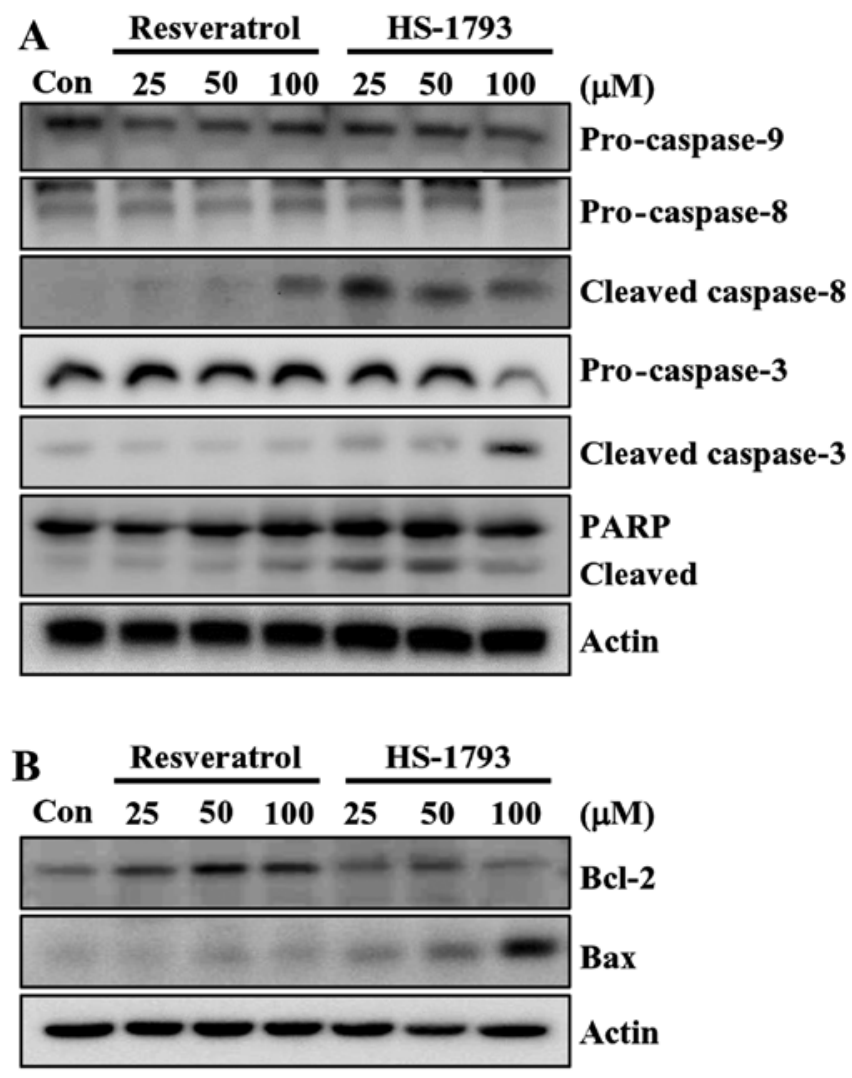

C

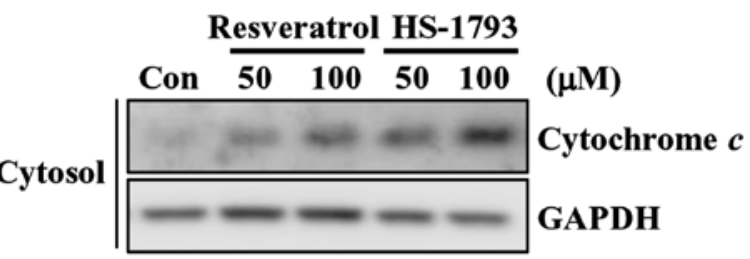

Mitochondria

Cytochrome $c$

GAPDH

Figure 4. HS-1793 increases apoptosis in HCT116 cells. (A and B) Cells were treated with different concentrations of resveratrol or HS-1793 for $24 \mathrm{~h}$ Whole-cell lysates were subjected to western blot to assess the expression of apoptosis-related proteins. Actin was used as an internal control. (C) The cytosolic and mitochondrial proteins were extracted and subjected to western blot analysis. GAPDH was used as an internal control for the cytosolic fraction. Data represent one of three independent experiments. Con, vehicletreated control.

expressions of cyclin B1 and Cdc25C in HCT116 cells but with different potency (Fig. 6A).

Recent report demonstrated that resveratrol induced G1/S-phase arrest in human colon carcinoma cells (28), we investigated whether resveratrol as well as HS-1793 affect the levels of proteins involved in G1/S phase arrest. Resveratrol and its analogue HS-1793 showed differential levels of downregulation of CDK2, CDK4 and CDK6 in HCT116 cells (Fig. 5B). HS-1793 at $25 \mu \mathrm{M}$ decreased levels of CDK4 while 50 and $100 \mu \mathrm{M}$ of resveratrol induced downregulation of CDK4. HS-1793 at 50 and $100 \mu \mathrm{M}$ reduced the level of CDK6 while a slight decrease of CDK6 was observed at $100 \mu \mathrm{M}$ resveratrol (Fig. 6B). Neither resveratrol nor HS-1793 altered the level of cyclin D1 and cyclin A in HCT116 cells (Fig. 6B).

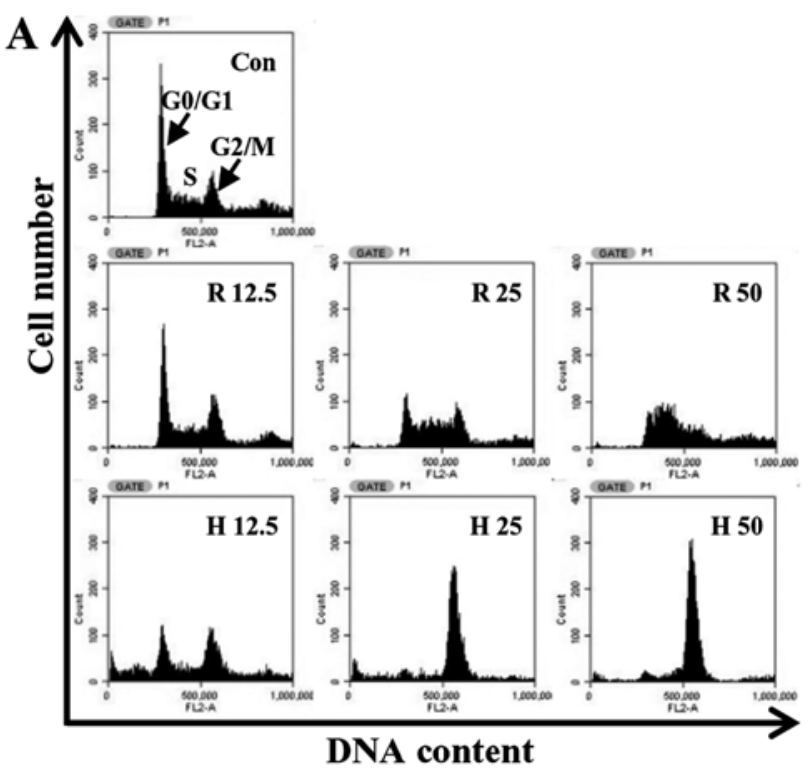

B

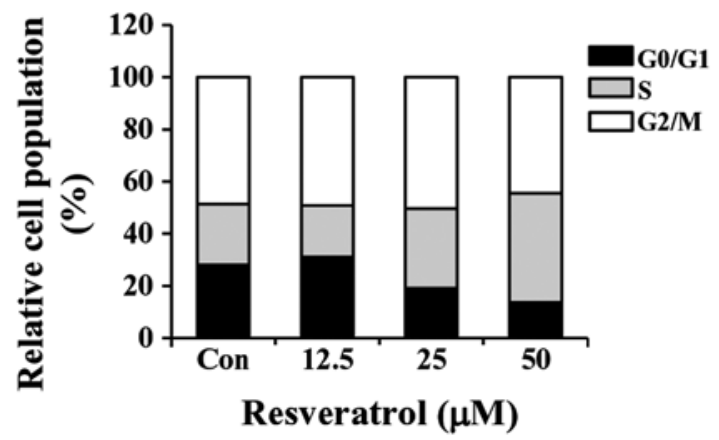

C

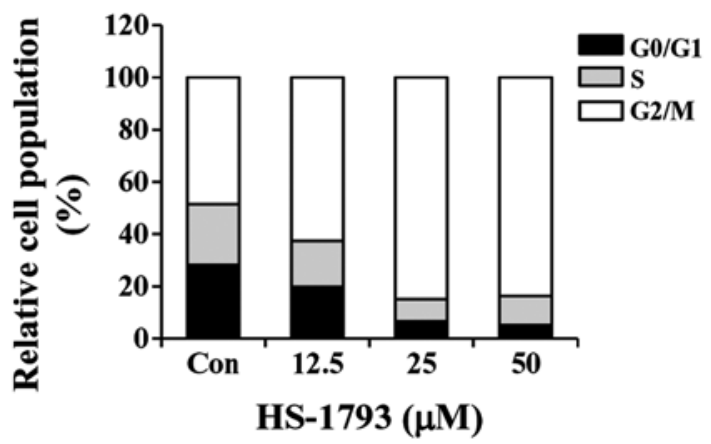

Figure 5. HS-1793 induces cell cycle arrest in HCT116 cells. (A) Cells were treated with different concentrations $(0,12.5,25$ and $50 \mu \mathrm{M})$ of resveratrol (R) or HS-1793 (H) for $24 \mathrm{~h}$, stained with PI, and then subjected to flow cytometry to measure their distribution in each phase of the cell cycle. Data represent one of three independent experiments. (B and C) Cell cycle distribution in (B) resveratrol- or (C) HS-1793-treated cells. Con, vehicle-treated control.

HS-1793 inhibits Akt and ERK phosphorylation in HCT116 cells. The AKT/protein kinase B (PKB) kinases have been shown to play critical roles in controlling the cellular processes including cell growth, proliferation, survival and apoptosis (29). Western blot analyses showed a significant decrease in Akt phosphorylation in cells treated with resveratrol or HS-1793 (Fig. 7A). The results also indicated that resveratrol did not affect total Akt level, while HS-1793 slightly reduced total Akt at high-concentration (Fig. 7A). We also investigated whether HS-1793 modulates the MAPK cascades including JNK, ERK1/2 and p38 MAPK. HS-1793 


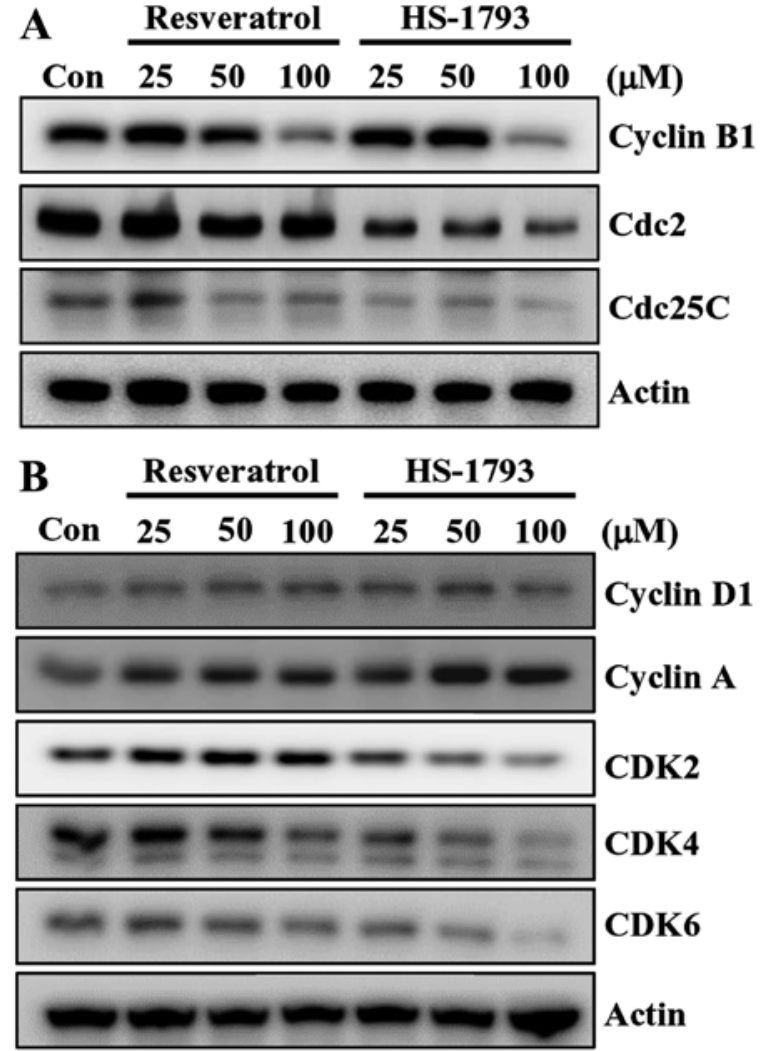

Figure 6. HS-1793 modulates expression of cell cycle modulatory proteins. Cells were treated with different concentrations $(0,25,50$ and $100 \mu \mathrm{M})$ of resveratrol or HS-1793 for $24 \mathrm{~h}$. Whole-cell lysates were subjected to western blot to assess the expression of (A) G2/M and (B) G1 regulatory proteins. Actin was used as an internal control. Data represent similar results from three independent experiments. Con, vehicle-treated control.

decreased the phosphorylation of ERK1/2 without affecting the protein level (Fig. 7A). HS-1793 was more potent than resveratrol in diminishing ERK1/2 phosphorylation. However, HS-1793 and resveratrol did not affect the activation of JNK and p38 MAPK.

To confirm the involvement of Akt signaling in apoptosis induced by HS-1793, we employed LY294002 to inactivate Akt, treating HCT116 cells with HS-1793. The results indicated that HS-1793 and LY294002 treatment alone induced 33 and $15 \%$ cell death, respectively. The MTT assay results also showed that enhanced apoptotic effects of HS-1793 on HCT116 cells were observed when co-treated with LY294002 in comparison to treatment with HS-1793 alone (Fig. 7B). Pharmacological inhibition of Akt with HS-1793 treatment increased levels of cleaved PARP compared with either treatment alone (Fig. 7C). Of note, treatment of HCT116 cells with LY294002 and HS-1793 was found to significantly suppress Akt activation. These results suggest that the Akt pathway is likely involved in HS-1793-induced growth inhibition and apoptosis of HCT116 cells.

\section{Discussion}

In the present study we evaluated and compared the anticancer activity of HS-1793 with resveratrol in human colon cancer cell line HCT116. The MTT assay revealed that HS-1793 is more potent than resveratrol in the inhibition of cell growth and

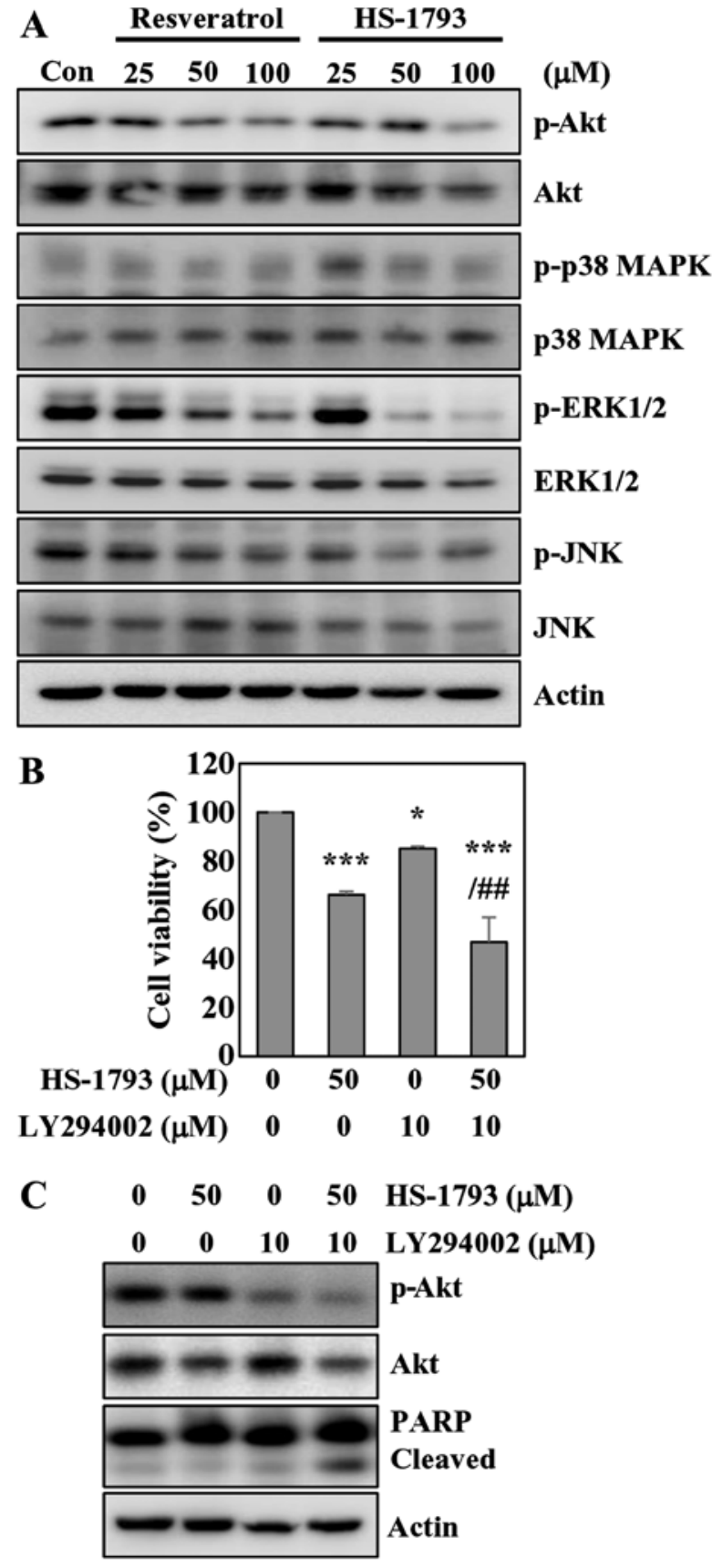

Figure 7. HS-1793 inhibits Akt and ERK activation. (A) Cells were treated with different concentrations $(0,25,50$ and $100 \mu \mathrm{M})$ of resveratrol or HS-1793 for $24 \mathrm{~h}$. Whole-cell lysates were subjected to western blotting to assess the level of Akt and MAPK pathway. Actin was used as an internal control. Data represent similar results from three independent experiments. (B) Cells were pretreated with LY294002 $(10 \mu \mathrm{M})$ for $30 \mathrm{~min}$, then treated with HS-1793 for $24 \mathrm{~h}$. MTT assay was performed to measure cell viability. Data are the mean $\pm \mathrm{SD}$ of three independent experiments. ${ }^{*} \mathrm{p}<0.05$ and ${ }^{* * *} \mathrm{p}<0.001$ vs. vehicle-treated cells; ${ }^{\# \#} \mathrm{p}<0.01$ vs. HS-1793-treated cells. (C) Cells were pretreated with LY294002 $(10 \mu \mathrm{M})$ for $30 \mathrm{~min}$ and subsequently treated with HS-1793 $(50 \mu \mathrm{M})$ for $24 \mathrm{~h}$. Cells were lysed and equal amounts of proteins were subjected to western blot to assess the expression of Akt and PARP cleavage. Actin was used as an internal control. Data represent similar results from three independent experiments. Con, vehicle-treated control.

proliferation in HCT116 cells. At equimolar concentrations, HS-1793 is also more potent than resveratrol in the induction of apoptotic cell death, evidenced by Annexin V staining, pro-caspase-3 reduction, cytochrome $c$ release and cleaved PARP, in colon cancer cells. Flow cytometric analysis indicated 
that resveratrol caused S phase arrest, whereas HS-1793 induced G2/M arrest in tested colon cancer cells. HS-1793 induced cell cycle progression mainly by downregulating cyclins and CDKs. In addition, we found that HS-1793 was substantially more potent than resveratrol at inhibition of Akt.

We found that HS-1793 was more effective in inhibiting cell growth and proliferation, and induced apoptosis in CRC cells. The observed growth inhibitory efficacy and apoptogenic cell death-inducing properties of HS-1793 are in agreement with those observed by ours and others in prostate cancer (30), breast cancer $(23,31,32)$, colon cancer (22) and leukemia (21). We reported previously that HS-1793 triggered apoptosis in two breast cancer cell lines by mediating p53-dependent and -independent pathways (23). The same study also described that HS-1793 was respectively, 2-fold more potent in inducing apoptotic cell death (23). In the present study, we confirmed that HS-1793 exhibited more potent anticancer property than resveratrol in HCT116 CRC cells, while HCT116 cells seem to be less sensitive to resveratrol and its analogue HS-1793 in response to that in breast cancer cells (23). The ability of HS-1793 to decrease the protein level of pro-caspases and the subsequent cleavage of PARP further supports the apoptogenic property of HS-1793 against CRC. We also found that HS-1793 upregulates the expression of pro-apoptotic Bax at the protein level. The Bcl-2 family proteins play critical roles in the induction of apoptosis. Indeed, the ratio between antiapoptotic Bcl-2 and apoptosis prompting Bax helps determine, in part, the susceptibility of cells to death signal (33). Although HS-1793 did not affect the protein level of Bcl-2, the change in $\mathrm{Bcl}-2 / \mathrm{Bax}$ ratio by HS-1793 is sufficient to induce apoptosis in HCT116 cells. The data here were consistent with the results of previous studies $(21,23,30)$, which suggested that HS-1793 had stronger antitumor effects than resveratrol and could induce cell death in part through the modulation of Bcl-2 family proteins.

Kim et al (23) showed that HS-1793 caused G2/M phase cell cycle arrest in the human breast cancer MCF-7 and MDA-MB-231 cells, and reduced the level of cell cycle regulatory proteins (cyclin $\mathrm{B} 1, \mathrm{Cdc} 2$ and $\mathrm{Cdc} 25 \mathrm{C}$ ) involve in G2/M. The present study observed that HS-1793 could induce G2/M phase cell cycle arrest in HCT116 cells. While resveratrol caused the accumulation of cells in the S phase in HCT116 cells. In addition, Liu et al (28) reported that resveratrol could inhibit proliferation of HCT116 cells by inducing G1/S phase cell cycle arrest, while we observed increased number of cells in $\mathrm{S}$ phase by resveratrol. The precise reason for this difference is not clear but conditions for the experiments may count for this discrepancy. Here, we found that resveratrol decreased the expression of CDK4 and CDK6 but not cyclin D1. The G1/S transition is regulated by complexes formed by cyclin $\mathrm{D}$ and its binding partners CDK4 or CDK6 (34). We also observed that HS-1793 markedly suppressed the expression of CDK4 and CDK6 although this resveratrol analogue caused G2/M arrest in colon cancer cells. Thus, HS-1793 was found to be more effective than resveratrol in inhibiting these two cyclin-dependent kinases. Therefore, it is likely that HS-1793 exerts its inhibitory effects on cancer cell cycle progression by modulating cell cycle regulator proteins, however, further mechanistic study is needed to elucidate the define mode of action of HS-1793.
Our result showed that HS-1793 inhibited the phosphorylation of Akt, which are involved in cancer cell growth and proliferation. In addition, we found that HS-1793 was substantially more potent than resveratrol at reduction of phosphorylated Akt. It has been shown that activation of Akt signaling pathway was frequently observed in patients with colon cancer $(35,36)$, and thus it has considered as therapeutic targets for cancer prevention (37). Our results are also consistent with another recent report that HS-1793 inhibits Akt activation in colon cancer cells (22).

Overall, our results suggest that HS-1793 exhibits antiproliferative and apoptosis-inducing effect in human colon cancer cells. A previous study demonstrated the role of endoplasmic reticulum stress and Akt on HS-1793-induced cell death in HT-29 colon cancer cells, however, the study did not provide the cell death mechanism on colon cancer cells in detail (22). Moreover, the anticancer ability from single cell line may give only limited information on an agent's biological response. Therefore, testing the activities in several cell lines are required to characterize and understand the mechanism of drug action, resistance and modulation. Thus, this study provides strong evidence to constitute significant advancement over the existing knowledge and this is needed before consequent in vivo preclinical study with HS-1793. Moreover, present study showed that HS-1793 is superior to its parental chemical resveratrol as a good candidate for novel anticancer agent. On the basis of these results, further studies are needed to confirm and extend the present study and pharmacokinetic/pharmacodynamics studies are required to use this novel resveratrol analogue HS-1793 as an anticancer agent.

\section{Acknowledgements}

This study was supported by the BasicScience Research Program through the National Research Foundation of Korea (NRF) funded by the Ministry of Education, Science and Technology (nos. 2012R1A1A2006753 and 2014R1A1A2055336). This study was also supported by the National Research Foundation of Korea (NRF) grant funded by the Korea government (MSIP) (no. 2009-0083538).

\section{References}

1. Siegel RL, Miller KD and Jemal A: Cancer statistics, 2015. CA Cancer J Clin 65: 5-29, 2015.

2. Jung KW, Won YJ, Kong HJ, Oh CM, Cho H, Lee DH and Lee KH: Cancer statistics in Korea: Incidence, mortality, survival, and prevalence in 2012. Cancer Res Treat 47: 127-141, 2015.

3. Shin A, Kim KZ, Jung KW, Park S, Won YJ, Kim J, Kim DY and Oh JH: Increasing trend of colorectal cancer incidence in Korea, 1999-2009. Cancer Res Treat 44: 219-226, 2012.

4. Li YH, Niu YB, Sun Y, Zhang F, Liu CX, Fan L and Mei QB Role of phytochemicals in colorectal cancer prevention. World $\mathrm{J}$ Gastroenterol 21: 9262-9272, 2015.

5. Bhat KP, Lantvit D, Christov K, Mehta RG, Moon RC and Pezzuto JM: Estrogenic and antiestrogenic properties of resveratrol in mammary tumor models. Cancer Res 61: 7456-7463, 2001.

6. Schneider Y, Duranton B, Gossé F, Schleiffer R, Seiler N and Raul F: Resveratrol inhibits intestinal tumorigenesis and modulates host-defense-related gene expression in an animal model of human familial adenomatous polyposis. Nutr Cancer 39: 102-107, 2001. 
7. Li ZG, Hong T, Shimada Y, Komoto I, Kawabe A, Ding Y, Kaganoi J, Hashimoto $Y$ and Imamura M: Suppression of $\mathrm{N}$-nitrosomethylbenzylamine (NMBA)-induced esophageal tumorigenesis in F344 rats by resveratrol. Carcinogenesis 23: 1531-1536, 2002.

8. Sale S, Tunstall RG, Ruparelia KC, Potter GA, Steward WP and Gescher AJ: Comparison of the effects of the chemopreventive agent resveratrol and its synthetic analog trans-3,4,5,4'-tetramethoxystilbene (DMU-212) on adenoma development in the $\mathrm{Apc}\left(\mathrm{Min}^{+}\right)$mouse and cyclooxygenase-2 in human-derived colon cancer cells. Int J Cancer 115: 194-201, 2005.

9. Liu HS, Pan CE, Yang W and Liu XM: Antitumor and immunomodulatory activity of resveratrol on experimentally implanted tumor of $\mathrm{H} 22$ in Balb/c mice. World J Gastroenterol 9: 1474-1476, 2003.

10. Chen Y, Tseng SH, Lai HS and Chen WJ: Resveratrol-induced cellular apoptosis and cell cycle arrest in neuroblastoma cells and antitumor effects on neuroblastoma in mice. Surgery 136: 57-66, 2004.

11. Pan MH, Gao JH, Lai CS, Wang YJ, Chen WM, Lo CY, Wang M, Dushenkov S and Ho CT: Antitumor activity of 3,5,4'-trimethoxystilbene in COLO 205 cells and xenografts in SCID mice. Mol Carcinog 47: 184-196, 2008.

12. Chen JC, Chen Y, Lin JH, Wu JM and Tseng SH: Resveratrol suppresses angiogenesis in gliomas: Evaluation by color Doppler ultrasound. Anticancer Res 26: 1237-1245, 2006

13. Busquets S, Ametller E, Fuster G, Olivan M, Raab V, Argilés JM and López-Soriano FJ: Resveratrol, a natural diphenol, reduces metastatic growth in an experimental cancer model. Cancer Lett 245: 144-148, 2007.

14. Kosmeder JW II, Pezzuto JM and Pezzuto JM; Bhat KP Biological effects of resveratrol. Antioxid Redox Signal 3: 1041-1064, 2001.

15. Harikumar KB and Aggarwal BB: Resveratrol: A multitargeted agent for age-associated chronic diseases. Cell Cycle 7: 1020-1035, 2008.

16. Baur JA and Sinclair DA: Therapeutic potential of resveratrol: The in vivo evidence. Nat Rev Drug Discov 5: 493-506, 2006.

17. Cai YJ, Wei QY, Fang JG, Yang L, Liu ZL, Wyche JH and Han Z: The 3,4-dihydroxyl groups are important for trans-resveratrol analogs to exhibit enhanced antioxidant and apoptotic activities. Anticancer Res 24: 999-1002, 2004.

18. Szekeres T, Fritzer-Szekeres M, Saiko P and Jäger W: Resveratrol and resveratrol analogues - structure-activity relationship. Pharm Res 27: 1042-1048, 2010.

19. Song S, Lee H, Jin Y, Ha YM, Bae S, Chung HY and Suh H: Syntheses of hydroxy substituted 2-phenyl-naphthalenes as inhibitors of tyrosinase. Bioorg Med Chem Lett 17: 461-464, 2007.

20. Jeong SH, Lee JS, Jeong NY, Kim TH, Yoo KS, Song S, Suh H, Kwon TK, Park BS and Yoo YH: A novel resveratrol analogue HS-1793 treatment overcomes the resistance conferred by Bcl-2 and is associated with the formation of mature PML nuclear bodies in renal clear cell carcinoma Caki-1 cells. Int J Oncol 35: 1353-1360, 2009

21. Jeong SH, Jo WS, Song S, Suh H, Seol SY, Leem SH, Kwon TK and Yoo YH: A novel resveratrol derivative, HS1793, overcomes the resistance conferred by Bcl-2 in human leukemic U937 cells. Biochem Pharmacol 77: 1337-1347, 2009.

22. Um HJ, Bae JH, Park JW, Suh H, Jeong NY, Yoo YH and Kwon TK: Differential effects of resveratrol and novel resveratrol derivative, HS-1793, on endoplasmic reticulum stress-mediated apoptosis and Akt inactivation. Int J Oncol 36: 1007-1013, 2010.
23. Kim JA, Kim DH, Hossain MA, Kim MY, Sung B, Yoon JH, Suh H, Jeong TC, Chung HY and Kim ND: HS-1793, a resveratrol analogue, induces cell cycle arrest and apoptotic cell death in human breast cancer cells. Int J Oncol 44: 473-480, 2014

24. Kim DH, Hossain MA, Kim MY, Kim JA, Yoon JH, Suh HS, Kim GY, Choi YH, Chung HY and Kim ND: A novel resveratrol analogue, HS-1793, inhibits hypoxia-induced HIF-1 $\alpha$ and VEGF expression, and migration in human prostate cancer cells. Int J Oncol 43: 1915-1924, 2013

25. Wang $\mathrm{C}$ and Youle RJ: The role of mitochondria in apoptosis. Annu Rev Genet 43: 95-118, 2009.

26. Sancar A, Lindsey-Boltz LA, Unsal-Kaçmaz K and Linn S: Molecular mechanisms of mammalian DNA repair and the DNA damage checkpoints. Annu Rev Biochem 73: 39-85, 2004

27. Perry JA and Kornbluth S: Cdc25 and Wee1: Analogous opposites? Cell Div 2: 12, 2007.

28. Liu B, Zhou Z, Zhou W, Liu J, Zhang Q, Xia J, Liu J, Chen N, Li M and Zhu R: Resveratrol inhibits proliferation in human colorectal carcinoma cells by inducing G1/S phase cell cycle arrest and apoptosis through caspase/cyclin CDK pathways. Mol Med Rep 10: 1697-1702, 2014.

29. Bellacosa A, Kumar CC, Di Cristofano A and Testa JR: Activation of AKT kinases in cancer: Implications for therapeutic targeting. Adv Cancer Res 94: 29-86, 2005.

30. Jeong NY, Yoon YG, Rho JH, Lee JS, Lee SY, Yoo KS, Song S, Suh $\mathrm{H}$, Choi YH and Yoo YH: The novel resveratrol analog HS-1793-induced polyploid LNCaP prostate cancer cells are vulnerable to downregulation of $\mathrm{Bcl}-\mathrm{xL}$. Int $\mathrm{J}$ Oncol 38: $1597-1604,2011$

31. Jeong SH, Song IS, Kim HK, Lee SR, Song S, Suh H, Yoon YG, Yoo YH, Kim N, Rhee BD, et al: An analogue of resveratrol HS-1793 exhibits anticancer activity against MCF-7 cells via inhibition of mitochondrial biogenesis gene expression. Mol Cells 34: 357-365, 2012

32. Kim HJ, Yang KM, Park YS, Choi YJ, Yun JH, Son CH, Suh HS, Jeong MH and Jo WS: The novel resveratrol analogue HS-1793 induces apoptosis via the mitochondrial pathway in murine breast cancer cells. Int J Oncol 41: 1628-1634, 2012.

33. Gross A, McDonnell JM and Korsmeyer SJ: BCL-2 family members and the mitochondria in apoptosis. Genes Dev 13 1899-1911, 1999.

34. Bates S, Bonetta L, MacAllan D, Parry D, Holder A, Dickson C and Peters G: CDK6 (PLSTIRE) and CDK4 (PSK-J3) are a distinct subset of the cyclin-dependent kinases that associate with cyclin D1. Oncogene 9: 71-79, 1994.

35. Malinowsky K, Nitsche U, Janssen KP, Bader FG, Späth C, Drecoll E, Keller G, Höfler H, Slotta-Huspenina J and Becker KF: Activation of the PI3K/AKT pathway correlates with prognosis in stage II colon cancer. Br J Cancer 110: 2081-2089, 2014.

36. Rychahou PG, Kang J, Gulhati P, Doan HQ, Chen LA, Xiao SY, Chung DH and Evers BM: Akt2 overexpression plays a critical role in the establishment of colorectal cancer metastasis. Proc Natl Acad Sci USA 105: 20315-20320, 2008.

37. Crowell JA, Steele VE and Fay JR: Targeting the AKT protein kinase for cancer chemoprevention. Mol Cancer Ther 6: 2139-2148, 2007. 